\title{
Growth-hormone Levels in Children and Adolescents
}

\author{
F. C. GREENWOOD*, M.SC., PH.D., W. M. HUNTER*, B.SC., \\ VALERIE J. MARRIAN $\dagger$, M.B., CH.B., M.R.C.P., D.C.H.
}

Brit. med. F., 1964, 1, 25-26

Growth hormone obtained from the pituitary gland was so termed because its biological effect in producing increases in body weight in experimental animals was readily measured. Changes in the secretion of human growth hormone from the pituitary and hence changes in plasma levels may be a major controlling factor of the changes in growth velocity shown to take place in children and adolescents (cf. Tanner, 1962). A previous attempt to correlate these changes (Girard, Vest, and Roth, 1961) was not successful, since the haemagglutination assay for human growth hormone in plasma yielded gross overestimates in the levels (cf. Wolstenholme and Cameron, 1962). The development of a specific and precise method for the assay of human growth hormone has enabled a further study of the problem to be made.

We have found that growth-hormone levels in individuals can fluctuate rapidly and considerably during the day. We have attempted to reduce this variation by standardizing the timing of plasma samples after meals to permit comparisons of growth-hormone levels in plasma between subjects of different ages. It is concluded that greater standardization and pituitaryfunction tests are required.

\section{Case Material and Methods}

The patients studied were obtained from a group of In-patients and out-patients at the Royal Hospital for Sick Children and Southmead Hospital, Bristol. The "normal" group consisted of children with a wide range of medical conditions, both psychosomatic and organic-for example, enuresis, constipation, respiratory infections, etc.-with no apparent endocrine disturbance. Ages ranged from birth to 14 years in 27 girls and from birth to 17 years in 41 boys. Two further groups of children showing disorders of growth were studied-a group of 14 children with small stature and a group of 15 obese children. These groups were selected by reference to the standard percentile charts for height and weight (Tanner and Whitehouse, 1959). Since it was not possible to obtain fasting samples of blood all specimens were taken from two to three hours after a meal. From 10 older children three or four blood samples were obtained during the course of a day, starting with a fasting sample. Aliquots $(0.2 \mathrm{ml}$.) for growth-hormone assay were obtained from heparinized plasma taken for other purposes and were stored and transported frozen.

The assay for growth hormone was carried out by the radioImmunoelectrophoretic method of Hunter and Greenwood (1962). The procedure used $0.2 \mu \mathrm{mg}$. of ${ }^{131} \mathrm{I}$-labelled human growth hormone and rabbit antiserum to human growth hormone $(1 / 200,000)$, and plasma was assayed at 0.05 to $0.4 \mathrm{ml}$. of 1/4-1/16 dilutions. The sensitivity of the assay is such that amounts of over $1 \mu \mathrm{mg}$. of growth hormone per ml. of plasma can be measured. Data on the specificity and precision of the assay are reported elsewhere (Hunter and Greenwood, 1964b).

\footnotetext{
- Scientific Staff, Imperial Cancer Research Fund, London.
}

+ Lecturer in Child Health, University of Bristol, Bristol 2.

\section{Results}

\section{Diurnal Variation of Human-growth-hormone Levels in Individual Subjects}

The results of serial assays for growth hormone over a ninehour period in five normal boys aged from 9 to $12 \frac{1}{2}$ years are shown in the Table together with estimates of sexual maturity. Of the five girls studied, aged from 11 to 14 years, three were past the menarche. The studies were not carried out as metabolic experiments with appropriate control of the intake

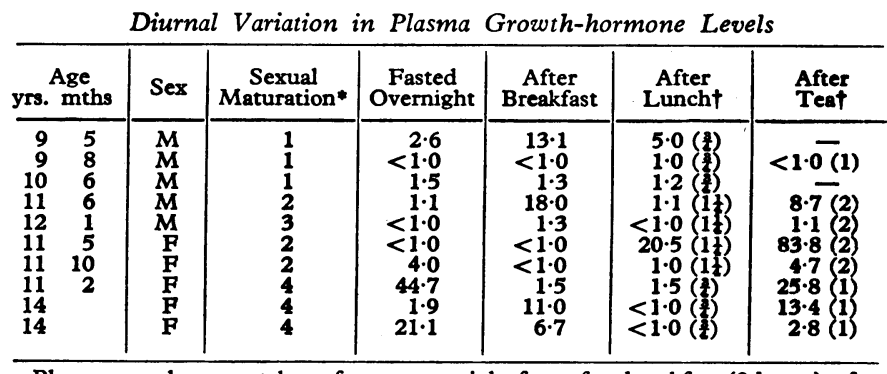

Plasma samples were taken after an overnight fast, after breakfast (2 hours), after lunch (three-quarters or one and a quarter hours), and after tea (one or two hours). mg. growth hormone per $\mathrm{ml}$. plasma.

* Tanner and Whitehouse (1959).

$\dagger$ Time in hours after meal are given in parentheses.

of food or sweets, but the results proved that growth-hormone levels can show rapid changes over short periods. It may he seen that in 11 of the 38 samples the level of human growth hormone was below the limit of sensitivity of the assay $(<1$ $\mu \mathrm{mg}$. growth hormone $/ \mathrm{ml}$. plasma). Where growth hormone was measured the levels showed considerable variation in an individual during the day (1-84 $\mu \mathrm{mg} . / \mathrm{ml}$.). This variation is not due to the method of assay, since over this range the measurements have a precision of \pm 0.08 to $\pm 2.2 \mu \mathrm{mg}$. (standard deviation). Such variation within individuals makes it impossible to compare the levels between individuals using single plasma samples taken at random during the day.

\section{Levels of Human Growth Hormone in Plasma Taken at Standard Times from Children Over a Wide Age Range}

Single blood samples from normal children (Figs. 1 and 2) were taken from two to three hours after a meal. The values for boys and girls were not different and are plotted separately only for clarity. There was no characteristic trend in values for growth hormone with age akin to the known changes in growth velocity. Either no such correspondence exists or the attempt to standardize conditions was inadequate to demonstrate the correlation. Over the age range some $64 \%$ of the values were above $3 \mu \mathrm{mg} . / \mathrm{ml}$. plasma. In contrast 18 adult values were below $3 \mu \mathrm{mg} . / \mathrm{ml}$. plasma, three falling between 1 and 3 $\mu \mathrm{mg}$. and the remainder below.

The mean level and standard deviation of the group of normal children and adolescents was $10.83 \pm 11.6 \mu \mathrm{mg}$. ; those of the adults taken under the same conditions were $0.55 \pm 0.68 \mu \mathrm{mg}$.

The values obtained in the group of obese children fall within the range of values for normals. The elucidation of any defect in growth-hormone secretion in this group would require the 
assessment of pituitary function under stimulation by hypoglycaemia. Children classified as of small stature on bone age and height and weight percentile also gave values in the normal range. Subsequent experience has shown that the absence of growth hormone in a fasting specimen only suggests a diagnosis of hypopituitarism. Confirmation or a measurement of the degree of pituitary impairment requires a pituitary stimulation test by a hypoglycaemia induced by tolbutamide (Hunter and Greenwood, 1964a).

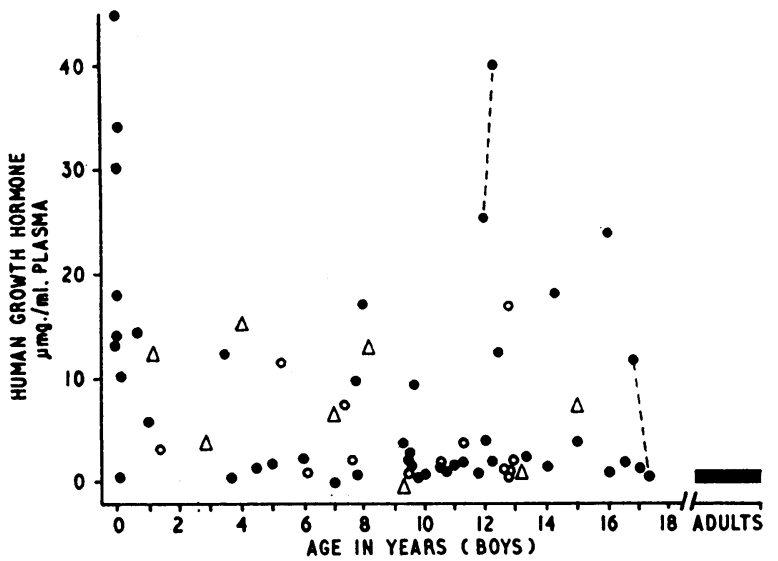

FIG. 1.-Plasma levels of growth hormone in boys. Blood samples were taken from two to three hours after a meal in normal boys $(O)$, or boys with small stature $(\triangle)$ or obesity $(O)$. Follow-up specimens are joined by a broken line. The range of plasma growth-hormone levels in 15 normal adults is shown.

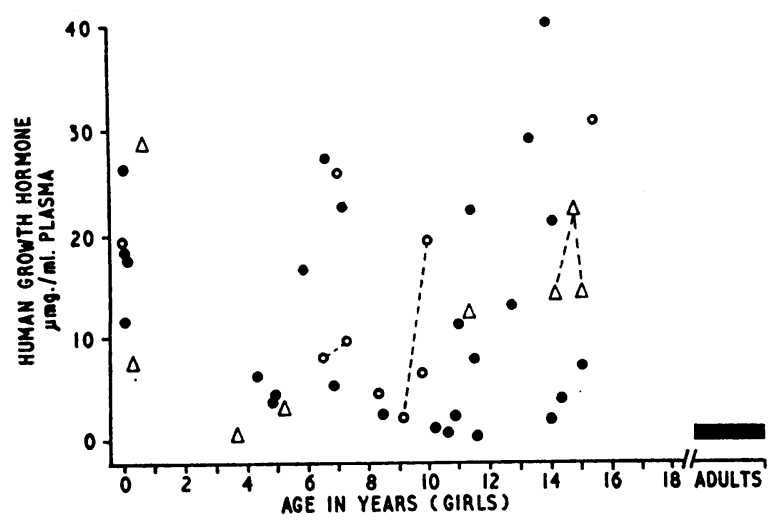

FIG. 2.-Plasma levels of growth hormone in girls. Application to girls of details given for Fig. 1 .

\section{Discussion}

The levels of growth hormone in a series of 42 children reported by Girard et al. (1961) using the haemagglutination assay are about 20 times higher than the values given by the present radio-immunoassay. The lack of specificity subsequently shown for this technique was no doubt responsible for the results obtained.

When the radio-immunoassay is used children and adolescents have approximately 20 times the amount of growth hormone found in plasma from adults, taken under similar conditions, two to three hours after a meal. The higher values in children compared with adults suggest that growth hormone may be a major component of the hormone complex controlling somatic growth in children. The lack of correlation between the level of growth hormone and age appears to be due to the difficulty of standardizing conditions for measuring a hormone shown to undergo rapid changes during the day.

It has recently been demonstrated that growth-hormone secretion in adults is stimulated by varying degrees of hypoglycaemia (Roth, Glick, Yalow, and Berson, 1963a). These authors report a fourfold increase in plasma growth hormone after a lengthy fast and a fiftyfold increase after the injection of insulin. Similar increases in adults have also been obtained after overnight fasting and exercise and after tolbutamide injection (Hunter and Greenwood, 1964a).

Further attempts to correlate growth-hormone levels with age in children and adolescents would seem to require the measurement of the levels of growth hormone and blood sugar after a standard fast. An alternative procedure, not possible in normal subjects, would be the measurement of the response of the pituitary to an acute hypoglycaemia induced by insulin or tolbutamide. The latter investigations would permit a study of the role of growth hormone as a factor in dwarfism, augmenting the systematic studies already available for adrenal, thyroid, pituitary, and pancreatic function. Obesity, like dwarfism, is also of complex aetiology, and it may be noted that tests of pituitary function in adult obese subjects (Roth, Glick, Yalow, and Berson, 1963b) have shown that growth-hormone secretion is not stimulated in obese subjects by fasting or exercise but is enhanced after an insulin-induced hypoglycaemia.

\section{Summary}

The level of circulating growth hormone in 68 children and adolescents has been measured, using a specific and precise radio-immunoelectrophoretic method for human growth hormone. Levels within an individual are shown to vary rapidly and considerably during the day. Plasma from children and adolescents taken two to three hours after a meal has about 20 times the concentration of growth hormone found in adult plasma taken under the same conditions. The values found in children and adolescents are not seen to correlate with age and hence growth velocity. Possible methods of demonstrating the expected correlation are outlined.

We wish to thank Dr. G. F. Marrian, F.R.S., for his encouragement of this work. We are grateful for the willing co-operation of the consultant paediatricians of the Royal Hospital for Sick Children and the Southmead Hospital, Bristol, who allowed us to study children in their care. The skilled technical assistance of Miss C. Harris and Mrs. H. Coats is gratefully acknowledged.

\section{REFERENCES}

Girard, J., Vest, M., and Roth, N. (1961). Nature (Lond.), 192, 1051

Hunter, W. M., and Greenwood, F. C. (1962). Biochem. 7., 85, 39P. - (1964a). To be published.

- (1964b). Biochem. F. In press.

Roth, J., Glick, S. M., Yalow, R. S., and Berson, S. A. (1963a). Science, 140, 987.

- (1963b). Metabolism, 12, 577.

Tanner, J. M. (1962). Growth at Adolescence, 2nd ed., Blackwell, Oxford.

- and Whitehouse, R. H. (1959). Lancet, 2, 1086.

Wolstenholme, G. E. W., and Cameron, M. P. (1962). Ciba Found. Coll. Endocr., 14, 45. 